\title{
Dynamic Range Compression by Differential Zone Mapping Based on Psychophysical Experiments
}

\author{
Francesco Banterle ${ }^{1 *}$ Alessandro Artusi ${ }^{2}$, Elena Sikudova $^{3}$, Thomas Bashford-Rogers ${ }^{4}$, \\ Patrick Ledda ${ }^{5}$, Marina Bloj ${ }^{6}$, and Alan Chalmers ${ }^{4}$ \\ ${ }^{1}$ Visual Computing Lab, ISTI-CNR, Italy, ${ }^{2}$ Departament d'Informtica i Matemtica Aplicada Universitat de Girona, Spain \\ ${ }^{3}$ Deapartment of applied informatics, Comenius University, Bratislava, Slovakia, \\ ${ }^{4}$ Visualisation Group, WMG, University of Warwick, UK, ${ }^{6}$ University of Bradford, UK, ${ }^{5}$ MPC Ltd, UK
}

\begin{abstract}
In this paper we present a new technique for the display of High Dynamic Range (HDR) images on Low Dynamic Range (LDR) displays. The described process has three stages. First, the input image is segmented into luminance zones. Second, the tone mapping operator (TMO) that performs better in each zone is automatically selected. Finally, the resulting tone mapping (TM) outputs for each zone are merged, generating the final LDR output image. To establish the TMO that performs better in each luminance zone we conducted a preliminary psychophysical experiment using a set of HDR images and six different TMOs. We validated our composite technique on several (new) HDR images and conducted a further psychophysical experiment, using an HDR display as reference, that establishes the advantages of our hybrid three-stage approach over a traditional individual TMO.
\end{abstract}

CR Categories: I.3.3 [Computer Graphics]: Picture/Image Generation Display Algorithms- [I.4.0]: Image Processing and Computer Vision-General Image Displays

Keywords: high dynamic range imaging, tone mapping, perceptual tone mapping

\section{Introduction}

The growth of the HDR imaging area has led to the development of many different TMOs that attempt to simulate different aspects of the Human Visual System (HVS). The intent is to solve issues, such as preserving local contrast, avoiding halo artifacts, simulating visual adaptation, enhancement and expansion of the original dynamic range, contrast reduction, image detail preservation etc. Figure 1 shows how two TMOs attempt to reproduce two different aspects such as the contrast and the detail. Figure $1(a)$ shows how the detail is well reproduced in the windows, but the contrast is not preserved. Figure $1(b)$ shows how the contrast is preserved in the bright and dark areas (see the white circles), but the details are not well reproduced (see the windows).

At the time of writing, two main problems remain unsolved. First,

\footnotetext{
*francesco.banterle@isti.cnr.it
}

choosing the proper TMO for a specific application can be difficult. Second, a comprehensive TMO that takes into account all aspects specified above has not yet been developed. Motivated by these two open issues and by the fact that developing a comprehensive TMO would be time consuming and a difficult task, we present a new technique for compressing HDR images into LDR display that takes into account the benefits of different existing TMOs and combines them, resulting in a more perceptually accurate image compared to the images obtained by existing TMOs. Figure 2 depicts our Framework. We decomposed, based on the luminance characteristics, the input HDR image into zones and analyzed the benefits of six different TMOs for each luminance zone. The TMOs that perform better in each luminance zone were selected. This step, based on human observations, and on the specific application where the TMOs are required to be applied, is able to choose the most appropriate TMO for the specific luminance zone. Finally, the results of the selected TMOs are combined to reproduce the final output LDR image. We demonstrate the results of our hybrid approach on several HDR images. Using an HDR display, showing the original HDR image as reference, we conducted a psychophysical experiment that clearly shows the advantages of our hybrid approach over using a traditional single TMO.

The rest of the paper is organized as follows: Section 2 describes related work. Section 3 presents our technique. Section 4 presents our results and subsequent validation. Finally, Section 5 concludes and presents future work.
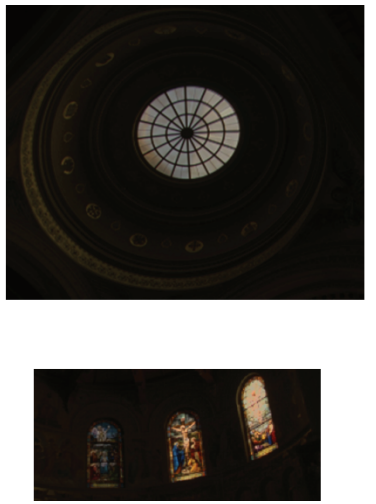

(a)

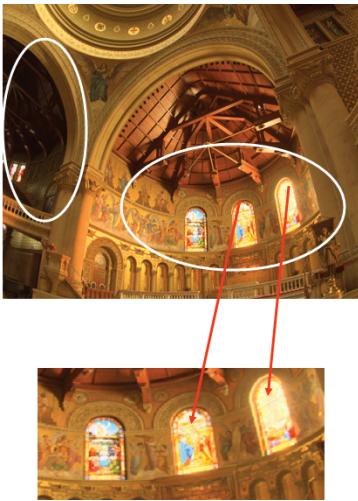

(b)
Figure 1: Example of two TMOs that take into account two different aspects. (a) The detail in the windows is well reproduced, but the contrast is not properly preserved. (b) The contrast in the bright and dark areas (white circles) is preserved but the detail, in the windows, is not well reproduced. The original image is copyright of Paul Debevec. 


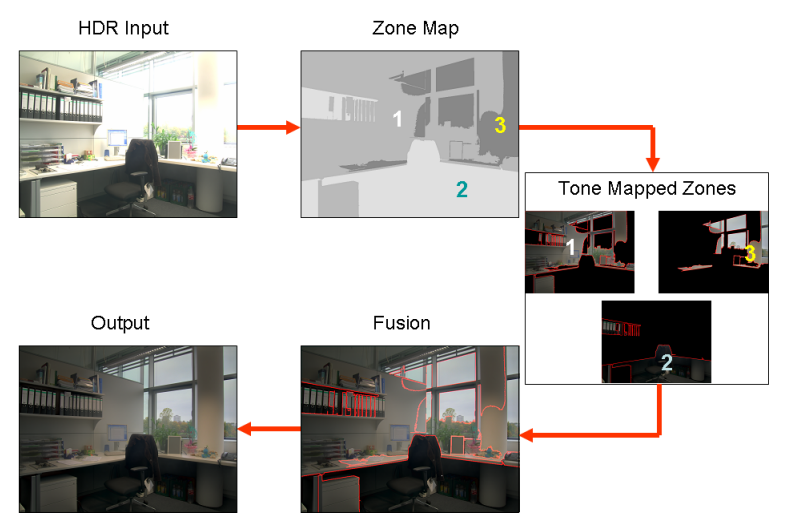

Figure 2: Schematic description of our Framework. First, the HDR input image is segmented into luminance zones and a zone map is created. Second, the optimal TMO for each zone is applied on the whole input image generating different output images (one for each zone). Finally the tone mapped images are merged and the final output is generated. (For visualization purposes we only show the specific zone tone mapped in the Tone Mapped Zones image on the right). The original image is copyright of Ahmet Oğuz Akyüz.

\section{Related Work}

The concept of tone mapping was introduced by Tumblin and Rushmeier [Tumblin and Rushmeier 1993], who proposed a tone reproduction operator that preserves the apparent brightness of scene features. Subsequently, many TMOs have been proposed. It is not the purpose of this paper to give a complete overview on HDR imaging and TM techniques. For a full overview see [Reinhard et al. 2010; Banterle et al. 2011b; Banterle et al. 2011a; Banterle et al. 2012a]. In this section, we will review only the approaches that are related to our work.

TMOs, that make use of segmentation to identify regions in the input image with common properties, are not new in the field of computer graphics. For example, the histogram adjustment proposed by Ward et al. [Ward Larson et al. 1997], was one of the first operators presented that belongs to this group of algorithms. This operator divides an image into bins and equalizes its histogram. However, it does not make use of the spatial information in the segmented areas for further compression of the dynamic range.

The model presented by Yee and Pattanaik [Yee and Pattanaik 2003] makes use of the spatial information. The HDR image is segmented into bins in the logarithmic space, pixels are labeled into groups, and the local adaptation luminance is calculated for each group. Finally, the local adaptation luminance is used to compress the dynamic range of the input image.

Krawczyk et al. [Krawczyk et al. 2005] proposed a perceptual approach that leverages the anchoring lightness perception theory [Gilchrist et al. 1999]. Their operator segments the image in frameworks, using a K-means approach, and calculates for each framework a weight map. After this step, for each framework the anchoring is calculated, and the frameworks are merged together using the weight maps. We have used a faster and simpler segmentation approach and a simple binary map in the fusion step. It is true that our segmentation approach may generate superficial boundaries between segments; but this problem is addressed by the use of our blending technique.

A user centered approach was investigated in Lischinski et al. [Lischinski et al. 2006]. In their method a soft segmentation is generated using rough brushes created by the user. Each brush determines the exposure that the user chose for the specific area. Then, the different exposures areas are merged to create the output image. The authors also proposed an automated initialization version of their approach, but user intervention is not completely eliminated due the interactive nature of their approach.

Mertens et al. [Mertens et al. 2007] presented an operator that can merge a stack of exposures without the generation of an HDR image. This method, for each exposure, generates a weight map of pixel well-exposed, which can be seen as a soft segmentation. Exposures are merged using these weight maps and Laplacian pyramids blending is adopted for avoiding seams. In our case, the weight map is a simple binary map and can be easily generated and further acceleration of our approach is straightforward (see Section 3.3).

Cadik [Cadik 2007] presented a perceptual motivated approach that is based on combination of arbitrary global and local tone mapping operators. This technique must carefully select the local and global TMOs in order to reproduce high quality output images and this selection is not based on human observation.

All the methods described do not adopt any human observation information for selecting different parameters/TMOs; they do not present any formal psychophysical validation and/or they rely on user intervention. The solution proposed in this paper, does not need user intervention, takes into account spatial information and is validated via a psychophysical study where the original HDR image is shown on an HDR display.

\section{Algorithm}

Our Framework, as illustrated in Figure 2, can be separated in three straightforward steps. Firstly, the input HDR image is segmented into luminance zones and a zone map is generated. Secondly, for each zone the most appropriate TMO is chosen and is applied on the whole input image generating different output images (one for each zone). In this way, the TMOs used in different zones are aligned to the same global parameters. Finally, the resulting outputs for each zone are merged. In the following subsections, each step is described in detail.

\subsection{Zone Map Generation}

The zone map is generated using the luminance information contained in the original HDR input image. First a segmentation step is performed. It separates the luminance (logarithmic scale) level into predefined zones, $\left(10^{i}, 10^{i+1}\right) \mathrm{cd} / \mathrm{m}^{2}$, and identifies to which zone the pixels of the input image belong. Once these preliminary zones are computed, additional steps are necessary. Firstly, the connections between the boundaries must be identified. This is done using a standard boundary continuity image processing algorithm, for details see [Gonzalez and Woods 2002].

Once the zones are properly segmented, small isolated areas are removed in order to avoid noise, Figure 3. This is done taking into account the number of pixels that belong to the specific zone. If the number of pixels belonging to a region is below $5 \%$ of the image size the region will be eliminated. This threshold value has been chosen based on the display resolution used in our experiments (HDR and LDR displays). Figure 3 shows that our approach is able to eliminate the noise regions resulting in a cleaner zone map. More sophisticated segmentation algorithms can be applied, for example we tried to use TurboPixels [Levinshtein et al. 2009], an approach for computing SuperPixels [Ren and Malik 2003]; the result of using this approach can be seen in the bottom images in Figure 3. The effort to use a more sophisticated approach during 
segmentation may produce better quality zones. However, in our experiments, we found out that extra computational efforts are not going to impact on the large areas but only at the boundaries of the zones, see Figure 3.

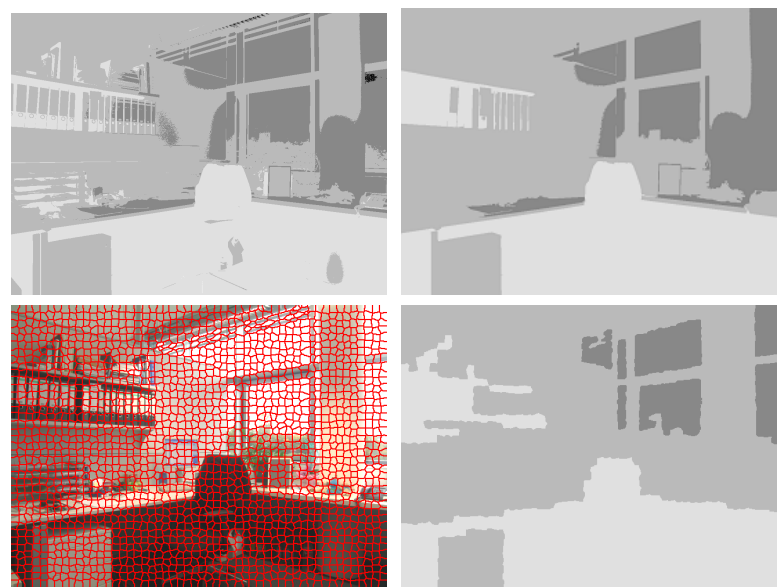

Figure 3: Zone map output: (top left) zone map with noise areas; (top right) the zone map as result of our threshold approach; (bottom left) segmentation of the HDR image using TurboPixels; (bottom right) zone map using TurboPixels after merging small TurboPixels. The original image is copyright of Ahmet Oğuz Akyüz.

Our method may lead to non optimal results in some cases. The rigid selection of the luminance threshold may produce superficial boundaries between segments. To avoid this problem a more sophisticated technique may be used, such as the one used in Krawczyk et al. [Krawczyk et al. 2005]. Any superficial boundaries are, however, removed afterwards anyway in the fusion step (see Section 3.3). Keeping this step simple enables a future straightforward implementation on a GPU.

\subsection{Tone Mapper Selection}

Several TMOs have been proposed in the last decade that attempt to achieve different goals and often it is difficult to either select the appropriate TMO for a specific goal, or to develop a comprehensive TMO that takes into account all these different aspects (Section 1). Our main goal is to show that is possible to select, based on human observations, a number of existing TMOs and use a combination of their results to reproduce an output image that takes into account all the advantageous aspects of the single TMOs. In order to identify which TMO performs better in a specific luminance zone, we conducted a series of psychophysical experiments.

To prove the concept above and be able to generalize its validity, we chose a subset of TMOs from the existing ones. These TMOs were selected based on the previous work by Ledda et al. [Ledda et al. 2005]. Therefore, in our work we investigated the following six TMOs: the algorithm proposed by Tumblin et al. [Tumblin et al. 1999]; the histogram model presented by Ward et al. [Ward Larson et al. 1997]; the time-dependent visual adaptation model [Pattanaik et al. 2000]; the photographic model (local operator) [Reinhard et al. 2002]; the bilateral filtering in the context of TM [Durand and Dorsey 2002]; and finally, the model presented by Drago et al. [Drago et al. 2003]. The TMOs are implemented as shown in [Reinhard et al. 2010], using the default parameters, as specified in [Reinhard et al. 2010], for each operator. In the case of the operator presented by Pattanaik et al. [Pattanaik et al. 2000], which takes into account the time dependency adaptation of the HVS, we used only the final frame of the adaptation process. We have chosen these operators based on their quality performances, usability and diffusion in the computer graphics community. We also limited the number of TMOs tested based on the fact that the purpose of this paper is to prove the concept and not to have a complete comparison of the qualities of the all existing TMOs in the computer graphics community. The iCAM method [Fairchild and Johnson 2002] was originally included however some of the generated images had a violet tinge which did not entirely disappear even adopting the solution suggested by the authors [Fairchild 2004].

\subsubsection{Set-up}

Once a luminance zone map is obtained, using the method described in subsection 3.1, the performance of the six TMOs in these luminance zones were compared psychophysically. We performed this test using a Dolby DR37-P HDR display [Dolby 2008] and two LDR LCD displays - which are identical to the front panel of the HDR display. All have a resolution of $1920 \times 1080$ pixels.

In these experiments, we used the set-up shown in Figure 5. The reference HDR image was presented on the HDR display (center). On the LDR display on the right, all of the LDR images obtained with the six TMOs together with the luminance zone map were displayed. By selecting one of the six images on this monitor, a full size version could be seen on the LDR display on the left. The specific luminance zone under examination was outlined in red on the luminance zone map and on both the HDR and full size LDR images. This outlining ensured that the observer was aware of the particular zone currently being evaluated. The participants could take as much time as they required to complete the task.

We used 15 HDR images with the characteristics reported in Table 1 and shown in Figure 4. The HDR images were reproduced with physically correct luminances following the method suggested in Akyüz et al. [Akyüz and Reinhard 2006]. Also in our case, the highlights that exceed the peak luminance of the HDR display were clipped around $3000 \mathrm{~cd} / \mathrm{m}^{2}$. Moreover, due to the flanking displays, a veiling effect can appear on the reproduced images. This problem was taken into account. A pilot experiment demonstrated that making use of a proper distance between each LDR and the HDR displays (half meter) avoided this problem.

\begin{tabular}{r|c|c} 
HDR Image & Resolution & Dynamic Range \\
\hline 1 & $1080 \times 721$ & 2.158 \\
2 & $1080 \times 1650$ & 3.496 \\
3 & $1080 \times 723$ & 2.675 \\
4 & $1080 \times 1440$ & 5.326 \\
5 & $1080 \times 810$ & 4.467 \\
6 & $1080 \times 1440$ & 3.389 \\
7 & $1080 \times 1440$ & 3.384 \\
8 & $1080 \times 1440$ & 3.932 \\
9 & $1080 \times 1440$ & 5.457 \\
10 & $1080 \times 1440$ & 5.903 \\
11 & $1080 \times 1619$ & 2.481 \\
12 & $1080 \times 1588$ & 2.827 \\
13 & $1080 \times 1621$ & 3.269 \\
14 & $1080 \times 1440$ & 3.266 \\
15 & $1080 \times 1646$ & 4.322
\end{tabular}

Table 1: Images used in the psychophysical experiment for the evaluation of the TMOs in the specific luminance zone. The dynamic range is expressed as order of magnitude.

The luminance zones available on the HDR display cover the range from $10^{-2}$ to $3 \times 10^{3} \mathrm{~cd} / \mathrm{m}^{2}$. The images, used in our experiments (see Table 1), cover the range from $10^{-2}$ to $10^{3} \mathrm{~cd} / \mathrm{m}^{2}$ as shown in the Table 2. The participants were asked to select the TMO that was considered to reproduce better results in a specified luminance zone. A group of 24 naive participants between 20 and 60 years 


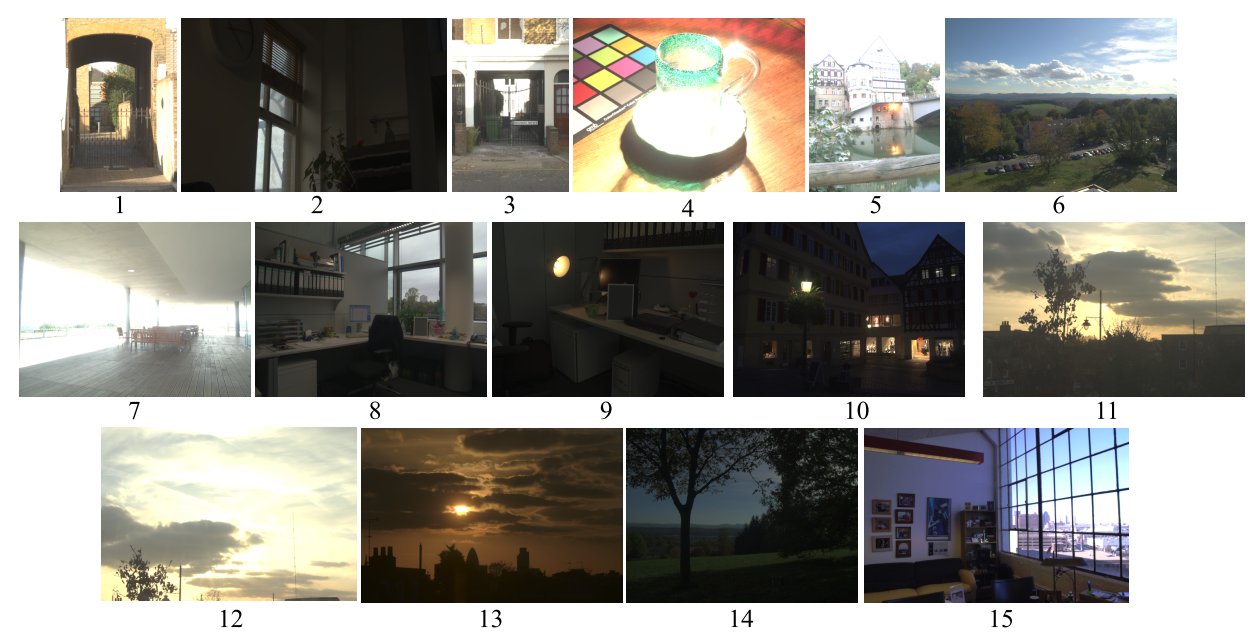

Figure 4: Images used in the psychophysical experiment. The number under the image corresponds to the numbers in the Table 1. Images 1-3 and 11-13 are copyright of Patrick Ledda. Images 4-10 and 14 are copyright of Ahmet Ŏguz Akyüz. Image 15 is copyright of Fredo Durand.

old with normal or corrected to normal vision took part in this experiment. The display was placed in a dark room minimizing the effects of ambient light. Prior to the start of the experiment, each participant was given five minutes to adapt to the environment.

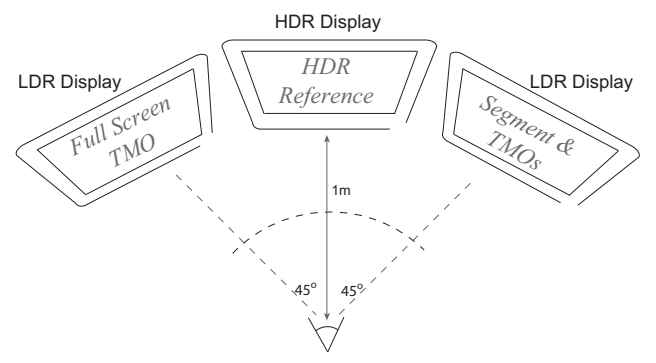

Figure 5: Set-up used in the psychophysical experiment evaluating which TMO performs best in each luminance zone. An HDR display is used as reference (center). The LDR display on the right is used to display the six TMOs used in the experiments and the luminance zone under examination. The LDR on the left is used to visualize full screen the TMO that the observer selected at each time in order to perform his/her evaluation.

\subsubsection{Data Analysis}

In our task, observers were asked to indicate for a given image and zone which of the six TMOs was closer to the reference. Since the observers did not rank all the algorithms we do not have ordinal data but rather categorical data. This allows us to use one-variable $\chi^{2}$ test to establish if the frequency distribution that we obtain (i.e the numbers on a row in Table 2) is significantly different from that which we would expect if participants showed no difference (all scores would be $100 / 6=16.6 \%$ ).

Each row corresponds to a luminance zone and each column to a TMO. The number in each cell indicates the percentage of times that a particular algorithm was chosen for a given image zone. The last column of the table reports the corresponding value of the test statistic $\chi^{2}$ for each image zone. In all cases $p<0.0001$ and the degree of freedom $d f=5$ (num. of categories - 1). In our case the number of categories is equal to the number of TMOs used in the experiment. Our results indicate that for all image zones observers chose an algorithm over others to be closer to the reference.

\begin{tabular}{r|c|c|c|c|c|c|c} 
Zone & 1 & 2 & 3 & 4 & 5 & 6 & $\chi^{2}$ \\
\hline 3 & $\mathbf{2 8 \%}$ & $22 \%$ & $23 \%$ & $1 \%$ & $20 \%$ & $6 \%$ & 78.23 \\
2 & $23 \%$ & $15 \%$ & $\mathbf{3 1 \%}$ & $1 \%$ & $20 \%$ & $10 \%$ & 98.24 \\
1 & $\mathbf{3 6 \%}$ & $6 \%$ & $32 \%$ & $1 \%$ & $14 \%$ & $11 \%$ & 138.4 \\
0 & $27 \%$ & $6 \%$ & $\mathbf{3 3 \%}$ & $1 \%$ & $22 \%$ & $11 \%$ & 72.40 \\
-1 & $\mathbf{5 2 \%}$ & $9 \%$ & $20 \%$ & $0 \%$ & $13 \%$ & $5 \%$ & 74.94 \\
-2 & $\mathbf{6 8 \%}$ & $0 \%$ & $6 \%$ & $0 \%$ & $26 \%$ & $0 \%$ & 98.35
\end{tabular}

Table 2: Statistical results of the psychophysical experiment evaluating which TMO performs best in each luminance zone. The enumeration corresponds to the followings TMOs: 1 - Drago et al., 2 - Pattanaik et al., 3 - Reinhard et al., 4 - Bilateral Filtering, 5 Ward et al., 6 - Tumblin et al. Drago et al. operator was ranked the best in the luminance zones $-2,-1,1$ and 3; and Reinhard et al. operator in the luminance zones 0 and 2.

In Table 2 the TMO that was most often selected for each zone is also highlighted in bold. The Drago et al. [Drago et al. 2003] operator was ranked the best operator in the luminance zones $-2,-1,1$ and 3; and the Reinhard et al. [Reinhard et al. 2002] operator in the luminance zones 0 and 2. Several TMOs evaluation works have been published lately [F. Drago and Seidel 2003], [Kuang et al. 2004], [Ledda et al. 2005], [Yoshida et al. 2005], [Cadik et al. 2006], [Akyüz and Reinhard 2006] and [Kuang et al. 2007]. The main difference of these works, in comparison with our experiment, is that the evaluation was done on the whole input images and not on a particular luminance zone. This can lead to a different evaluation since the subject, in these other experiments, can be influenced by the overall quality of the input image. In our case this is less evident since the subject is evaluating the TMO in a specific luminance zone without paying attention to the other areas of the input image. Our results in fact confirm, either partially or completely, previous TMOs evaluation works [Yoshida et al. 2005], [F. Drago and Seidel 2003], [Cadik et al. 2006]. However, the bilateral filter in our experiment was not performing well, as judged by our subjects, and this is partially in contradiction with the study presented by [Kuang et al. 2007] and [Akyüz and Reinhard 2006]. This can be explained by the fact that the original bilateral filtering presented by [Durand and Dorsey 2002] was modified in the works of [Kuang et al. 2007] and [Kuang et al. 2004] resulting in an improvement of the overall image contrast. In addition, [Kuang et al. 2007] and [Kuang et al. 2004] agree with our result that Reinhard et al. [Reinhard et al. 2002] was always well ranked. 
Concerning the work of Akyuz et al. [Akyüz and Reinhard 2006] we have noticed that the number of images used is $33 \%$ less than what has been used in our experiment and this may lead to different results. Also [Akyüz and Reinhard 2006] is in contradiction not only with our results, but also with the results of the others that confirm, either partially or completely, our results such as [Yoshida et al. 2005], [F. Drago and Seidel 2003], [Cadik et al. 2006], [Kuang et al. 2007], and [Kuang et al. 2004].

\subsection{Fusion Results}

Following the results of the previous Section we concluded that the Drago et al. [Drago et al. 2003] and the Reinhard et al. [Reinhard et al. 2002] operators would be utilized in the fusion step. Once the TMO to be used in each zone has been selected, the different TMOs are applied to the relevant zone and the outputs are blended to create the whole image. The blending operation is necessary to eliminate discontinuities in the luminance, that may occur at the edges of the zones within the map. A naïve blending of TMOs, is a simple multiplication of a weight map times the TMO with a consequent accumulation. This method creates artifacts such as seams and discontinuity at edges. To avoid these problems we blend the various TMOs using Laplacian blending, similar to that used in [Mertens et al. 2007].

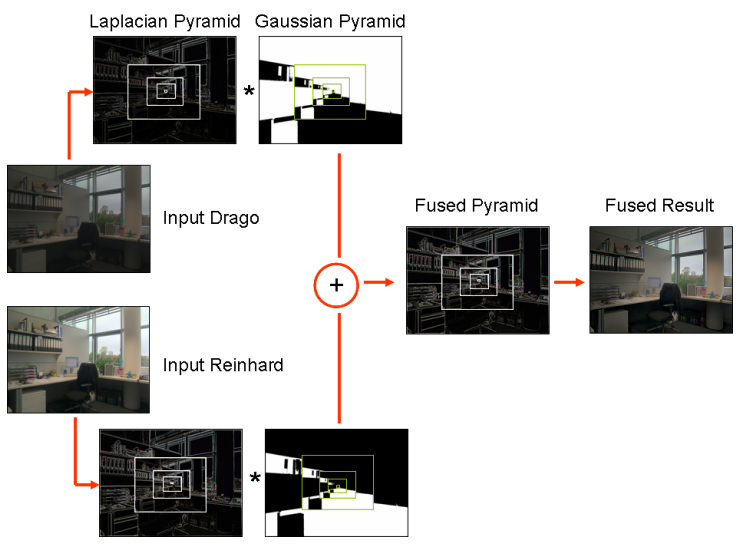

Figure 6: Graphical representation of the fusion step of our Framework. The original is copyright of Ahmet Oğuz Akyüz.

Figure 6 shows the graphical description of the fusion step. First, a Laplacian pyramid of the best performing TMOs is computed. Second, the Laplacian pyramid is multiplied by the Gaussian pyramid of the weight map associated with each TMO. Third, these results are summed and the fusion pyramid is generated. Finally, from the fusion pyramid the output image is reconstructed (Fused Result).

Based on the fact that the subjects' evaluation of the TMOs in the specific luminance zone, in the previous step Section 3.2, is equivalent to giving the same weight to all the pixels of the zone, we decided to use a simple Gaussian filtered binary map. We also investigated if a simple linear combination fusion scheme was able to give comparable results to the one obtained with the proposed fusion scheme. As is shown in Figure 7, several artifacts are visible in the output obtained with the simple fusion scheme (left image).

\section{Results}

In this Section we present the experimental results obtained from testing our hybrid approach.
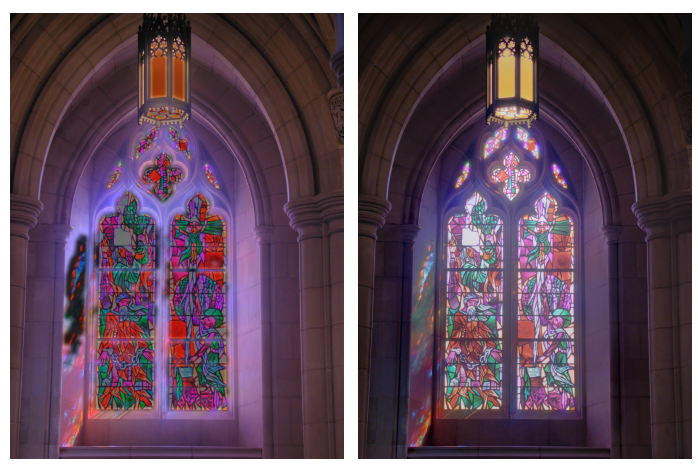

Figure 7: Comparison of the results obtained with two different fusion schemes: (left) image obtained with the simple fusion scheme, (right) and with the proposed fusion scheme. Artifacts are visible on the image obtained with the simple fusion scheme (left). The original image is copyright of Max Lyons.

\subsection{Set-up}

We tested and psychophysically validated our hybrid approach on several HDR images, using the same HDR and LDR displays as in the previous experiment. Again the HDR display was used to visualize the HDR image as the reference. We conducted a pairwise comparison test between the two best TMOs (Drago et al. and Reinhard et al.) and our hybrid operator. Figure 8 shows the set-up for this experiment; the reference HDR image is displayed on the HDR display (center), and the two TMOs that are being compared are displayed on the two LDR displays. This is a similar approach to evaluating TMOs as used in [Ledda et al. 2005].

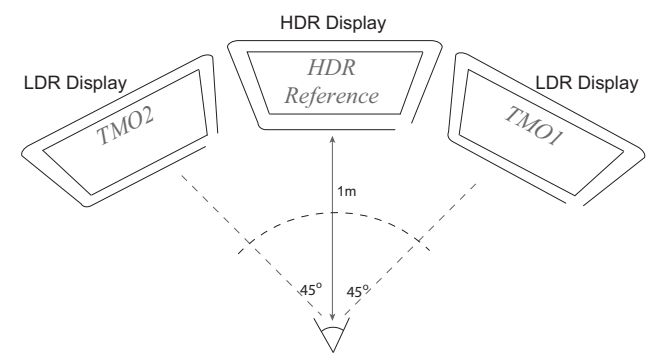

Figure 8: Set-up used in the validation experiment done for evaluating our hybrid TMO versus the two best TMOs of the previous psychophysical experiment. An HDR display is used as reference (center). Two LDR displays (right and left) are used for pairwise comparison.

We tested 13 images, different from the ones used in the previous experiment, resulting in 39 possible pair comparisons for each participant (3 possible pairs $\times 13$ images). A group of 21 naive participants between 20 and 60 years old with normal or corrected to normal vision took part in this experiment. Environmental conditions were the same as the previous TMO selection experiment. The characteristics of the 13 HDR images, used in this experiment, are reported in Table 3 and shown in Figure 9.

\subsection{Data Analysis}

In order to determine which TMO performs best, observers were asked to select, for each pair of images, the TMO that was considered to reproduce the result perceptually closer to the HDR reference image. We used a Multiple Comparison Range test, for this 

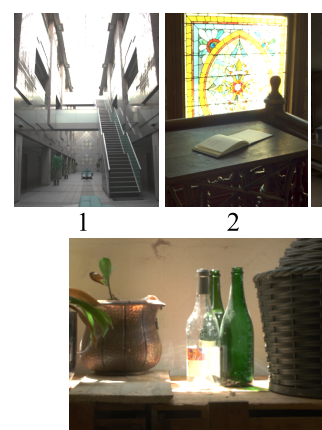

8
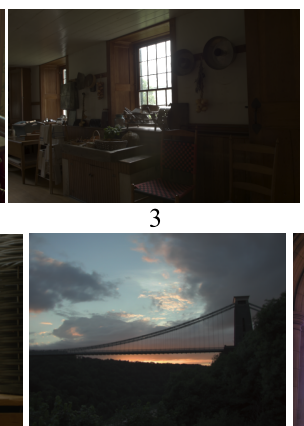

9
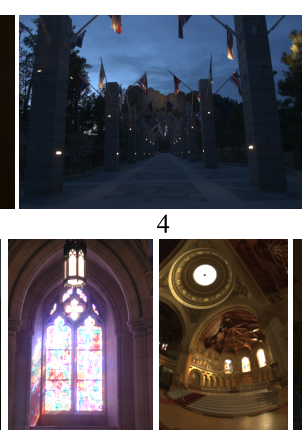

10

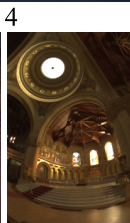

11

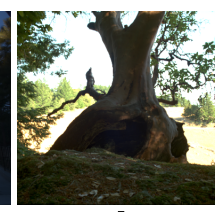

12
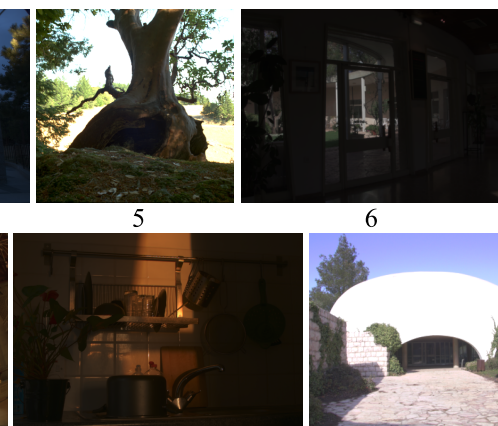

6

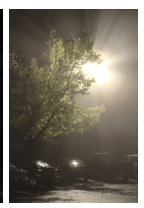

7

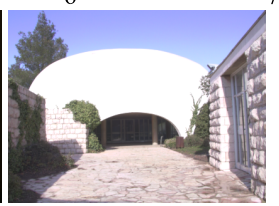

Figure 9: Images used in the psychophysical experiment; the number under the image corresponds to the numbers in Table 3. Image 1 is copyright of Karol Myszkowski. Images 2 and 5 are copyright of ILM Ltd. Images 3-4 are copyright of Mark Fairchild. Image 6 and 13 are copyright of Raanan Fattal. Image 7 is copyright of Jack Tumblin. Image 8 is copyright of Francesco Banterle. Image 9 is copyright of Greg Ward. Image 10 is copyright of Max Lyons. Image 11 is copyright of Paul Debevec. Image 12 is copyright of Patrick Ledda.

\begin{tabular}{r|c|c} 
HDR Image & Resolution & Dynamic Range \\
\hline 1 & $1016 \times 760$ & 4.3411 \\
2 & $874 \times 644$ & 5.8152 \\
3 & $1080 \times 1626$ & 5.1332 \\
4 & $1080 \times 1626$ & 4.6884 \\
5 & $906 \times 928$ & 4.334 \\
6 & $769 \times 1025$ & 5.0019 \\
7 & $1080 \times 718$ & 4.0791 \\
8 & $684 \times 912$ & 5.0932 \\
9 & $768 \times 1024$ & 3.8597 \\
10 & $1023 \times 767$ & 4.5301 \\
11 & $768 \times 512$ & 5.4024 \\
12 & $1080 \times 1624$ & 4.0761 \\
13 & $769 \times 1025$ & 2.4516
\end{tabular}

Table 3: Images used in the psychophysical experiment for the evaluation of our $T M O$. The dynamic range is expressed as order of magnitude and it is covering the same dynamic range as the images used in the first experiment.

\begin{tabular}{l|c|c|c} 
& $1^{\text {st }}$ & $2^{\text {nd }}$ & $3^{\text {rd }}$ \\
\hline Image 1 & HYB 26 & PHO 20 & DRA 17 \\
Image 2 & DRA 37 & HYB 25 & PHO 1 \\
Image 3 & HYB 38 & DRA 20 & PHO 5 \\
Image 4 & PHO 30 & DRA 18 & HYB 15 \\
Image 5 & HYB 30 & DRA 27 & PHO 5 \\
Image 6 & HYB 26 & DRA 23 & PHO 14 \\
Image 7 & HYB 34 & DRA 24 & PHO 5 \\
Image 8 & DRA 35 & HYB 23 & PHO 5 \\
Image 9 & HYB 30 & PHO 19 & DRA 14 \\
Image 10 & HYB 38 & DRA 14 & PHO 11 \\
Image 11 & DRA 34 & HYB 24 & PHO 5 \\
Image 12 & HYB 31 & DRA 24 & PHO 8 \\
Image 13 & HYB 30 & DRA 26 & PHO 7 \\
& & & \\
\hline Overall & HYB 370 & DRA 298 & PHO 150
\end{tabular}

Table 4: Results of the pairwise validation test. Each row represents the results for each image used in the experiment. The row at bottom is the overall result. The columns represent the $1^{\text {st }}, 2^{\text {nd }}$ and $3^{\text {rd }}$ TMO classified respectively. The number beside each operator represents the number of times it was chosen. The notation is the following: HYB is the Hybrid TMO presented in this paper, DRA is the Drago et al. and PHO is the Reinhard et al. Photographic operator.

paired-comparison experiment to determine if any pairwise difference was significant. This series of tests determines if inconsistencies in the scores between any of the two TMOs are present. This procedure, equivalent to Tukey's method used with ANOVA [David 1988], is based on the range of the scores obtained by the three TMOs. In this test, the difference between the scores of two TMOs is considered significant if it is greater than a critical value, $R$ [David 1988]. In other words $R$ represents the minimum value where the difference between the scores of two TMOs is consider non-significant.

We have chosen the significance level $\alpha$ equal to 0.05 and the critical values $R$ is computed as:

$$
R=\frac{1}{2} W_{t}, \alpha \sqrt{s t}+\frac{1}{4}
$$

where $W_{t},{ }_{\alpha}$ is the upper significance point of the $W_{t}$ distribution. At a significance level of 0.05 and for three TMOs $\left(t=3\right.$ the $W_{t}, \alpha$ is equal to 3.31 (see Pearson [Pearson and Hartley 1988], table 22). The value of $s$ specifies the total number of possible cases in the experiment. Table 4 shows the results of the second experiment.

We performed the multiple comparison range test on the results of the second experiment, for each image individually ( $s=$ num. operators) and for the combined preference over all images $(s=$ num. operators $\times$ num. images) This test shows that overall the hybrid TMO performs significantly better than either of the other two TMOs used on their own. The critical value $R$ is 48 when evaluated over all images. Since the difference in overall scores between each TMO is greater than $R$, the differences can be considered significant.

The test results show that the hybrid operator is not significantly worse for any of the test images than the other two operators. In the cases when Reinhard et al. or Dargo et al. operators appear to have higher scores the difference is less than the critical value of $R$, which for the case of single images is 14 .

Figure 10 shows some results: (first row) our Hybrid, (second row) Drago et al. and (third row) Reinhard et al. operator.

\section{Conclusions and Future Work}

We have presented a novel approach consisting of an hybrid TMO that tackles the problem that existing TMOs do not achieve similar quality results on different luminance zones of an image. Combining existing TMOs in a selective fashion enables us to take advantage of just the best properties of existing TMOs and hence produce images that human observers more often rank as closer to a reference HDR image. 

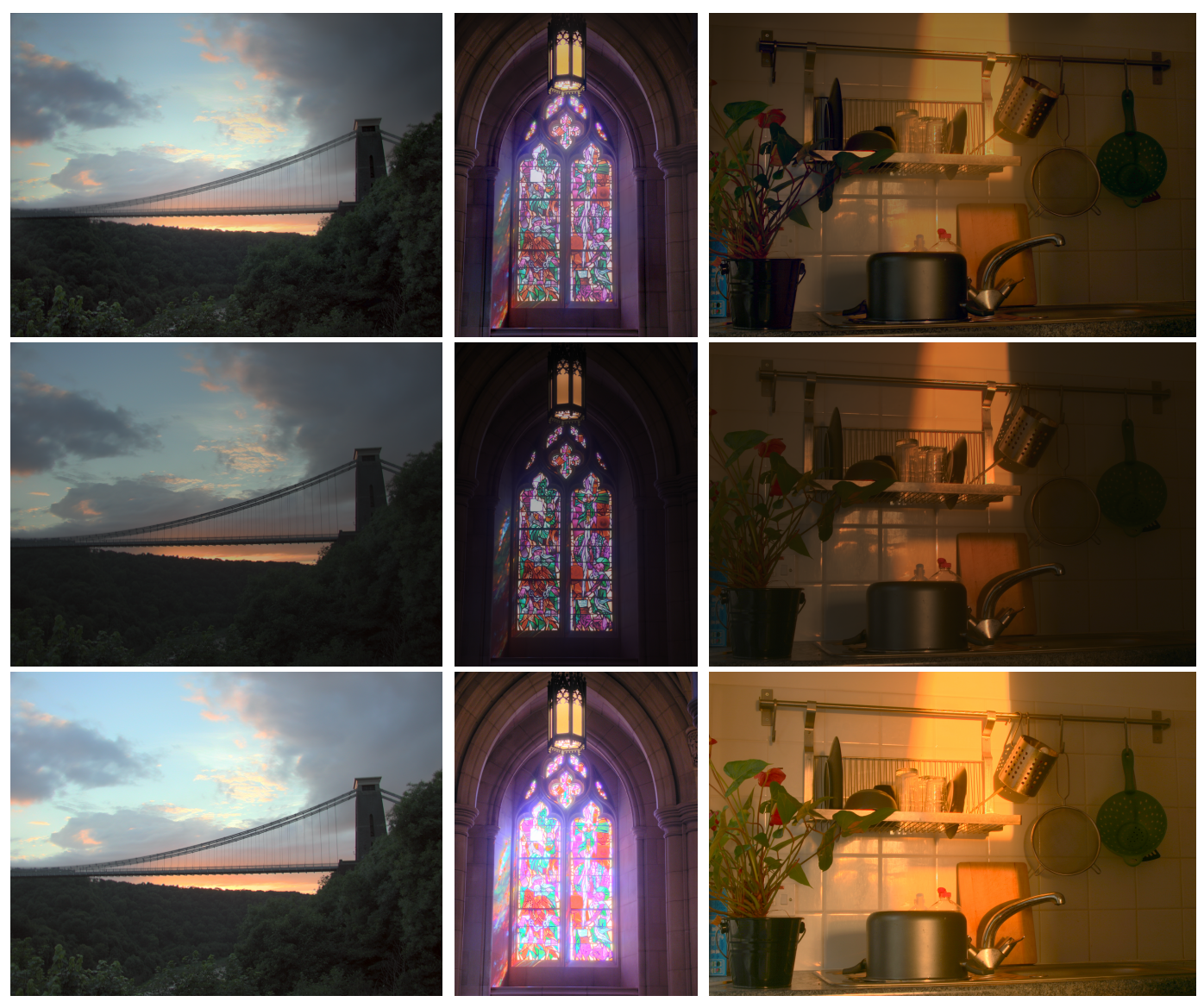

Figure 10: Results: (first row) our Hybrid, (second row) Drago et al. and (third row) Reinhard et al. operator. The original image on the left column is copyright of Greg Ward. The original image in the center is copyright of Max Lyons. The original image on the right column is copyright of Patrick Ledda.

Such a hybrid approach helps to address two of the open issues in the area of HDR imaging discussed in Section 1. First, based on psychophysical experiment we are able to determine which existing TMOs are more suitable for a specific application. Second, the resulting hybrid TMO will be comprehensive that takes into account all the important aspects of the selected existing TMOs.

Our approach has been validated on numerous HDR images through a detailed psychophysical experiment, using an HDR display as the reference. We also confirmed the statistical significance of the performance of the hybrid compared to the two best single TMOs.

Future work will extend this differential zone mapping approach for being used in HDR video applications and will implement a hardware solution to achieve real-time performances using Artusi et al. framework [Artusi et al. 2011] for speeding up Reinhard et al.'s operator [Reinhard et al. 2002]. This framework automatically detects the high contrast and textured regions in an HDR image and directs the highly computational demanding operation in these areas, while using a global tone mapping in the other regions. An alternative approach for speeding up Reinhard et al.'s operator would be to approximate the local part using a real-time bilateral filter [Banterle et al. 2012b]. Another interesting direction would be to apply the proposed approach to inverse tone mapping (ITM) [Banterle et al. 2009]. Many ITM operators have been proposed, they all have strength and weak points depending on which kind of image is applied to, i.e. well-exposed, over-exposed, very over-exposed, and under-exposed images. Therefore, the future direction would be to explore the level of exposedness of the image in order to understand which operator is more suitable for each level and to apply a different ITM operator to each exposedness level.

\section{Acknowledgements}

We thank Greg Ward, Ahmet Oğuz Akyüz, Max Lyons, Raanan Fattal, Karol Myszkowski, Jack Tumblin, Fredo Durand, Paul Debevec, Mark Fairchild, and ILM Ltd for HDR images used in our paper. We thank Gianpaolo Palma and Daniele Bernabei for their help in fixing the additional material and video. We thank anonymous reviewers for their comments. The work in this paper has formed part of EPSRC grant EP/D032148. Authors are funded by the following projects: the EC IST IP project 3D-COFORM (IST2008-231809), Ramon y Cajal amb referncia RYC-2011-09372, and VEGA $1 / 0602 / 11$. This work was partially supported by the European COST Action IC-1005.

\section{References}

AKYÜZ, A. O., AND ReInhard, E. 2006. Color appearance in high-dynamic-range imaging. SPIE Journal of Electronic Imaging 15, 3, 033001-1-033001-12.

Artusi, A., Michael, D., Roch, B., Chrysanthou, Y., And Chalmers, A. 2011. A selective approach for tone mapping 
high dynamic range content. In SIGGRAPH Asia 2011 Posters, ACM, New York, NY, USA, SA '11, 50:1-50:1.

Banterle, F., Debattista, K., Artusi, A., Pattanaik, S., Myszkowski, K., LeddA, P., AND Chalmers, A. 2009. High Dynamic Range Imaging and LDR Expansion For Generating HDR Content. Computer Graphics Forum 28, 8 (December).

Banterle, F., Artusi, A., Aydin, T. O., Didyk, P., Eisemann, E., Gutierrez, D., Mantiuk, R., AND MYSZKOWSKI, K. 2011. Multidimensional image retargeting. In SIGGRAPH Asia 2011 Courses, ACM, New York, NY, USA, SA '11, 15:1-15:612.

Banterle, F., Artusi, A., Debattista, K., And Chalmers, A. 2011. Advanced High Dynamic Range Imaging: Theory and Practice. AK Peters (CRC Press), Natick, MA, USA.

Banterle, F., Artusi, A., Aydin, T. O., Didyk, P., EiseMANn, E., Gutierrez, D., Mantiuk, R., AND Ritschel, T. 2012. Mapping images to target devices: spatial, temporal, stereo, tone, and color. In Eurographics 2012 Tutorials, Eurographics Association, EG '12.

Banterle, F., Corsini, M., Cignoni, P., And Scopigno, R. 2012. A low-memory, straightforward and fast bilateral filter through subsampling in spatial domain. Computer Graphics Forum 31, 1 (February), 19-32.

Cadik, M., Wimmer, M., Neumann, L., and Artusi, A. 2006. Image attributes and quality for evaluation of tone mapping operators. In Proceedings of the 14th Pacific Conference on Computer Graphics and Applications, National Taiwan University Press, Taipei, Taiwan, 35- 44.

CADIK, M. 2007. Perception motivated hybrid approach to tone mapping. Proceedings WSCG 2007.

DAVID, H. A. 1988. The Method of Paired Comparisons, 2nd ed. Oxford University Press.

Dolby. $2008 . \quad$ Dolby-DR37P. http://www.dolby.com/promo/hdr/technology.html (December).

Drago, F., Myszkowski, K., Annen, T., And Chiba, N. 2003. Adaptive logarithmic mapping for displaying high contrast scenes. Computer Graphics Forum (Proceedings of Eurographics '03).

DURAND, F., AND DORSEY, J. 2002. Fast bilateral filtering for the display of high dynamic range image. In Proceedings of SIGGRAPH 2002, 257-265.

F. Drago, W. L. Martens, K. M., And Seidel, H.-P. 2003. Perceptual evaluation of tone mapping operators. In Proceedings of the SIGGRAPH 2003 conference on Sketches and applications.

FAirchild, M., And Johnson, G. 2002. Meet icam: A nextgeneration color appearance model.

FAIRCHILD, M., $2004 . \quad$ MEET ICAM. http://www.cis.rit.edu/mcsl/icam/.

Gilchrist, A., Kossyfidis, C., Bonato, F., Agostini, T., Cataliotti, J., Li, X., Spehar, B., Annan, V., And ECONOMOU, E. 1999. An anchoring theory of lightness perception. Psychological Review 106, 4 (October), 795-834.

Gonzalez, R. C., And Woods, R. E. 2002. Digital image processing, 2. ed. ed. Prentice-Hall.
KrawczyK, G., Myszkowski, K., And Seidel, H.-P. 2005. Lightness perception in tone reproduction for high dynamic range images. In The European Association for Computer Graphics 26th Annual Conference EUROGRAPHICS 2005, Blackwell, Dublin, Ireland, vol. 24 of Computer Graphics Forum.

Kuang, J., Yamaguchi, H., Liu, C., Johnson, G. M., And FAIRCHILD, M. D. 2004. Evaluating hdr rendering algorithms. Color Image Conference, 315-320.

Kuang, J., Yamaguchi, H., Liu, C., Johnson, G. M., And FAIRCHILD, M. D. 2007. Evaluating hdr rendering algorithms. ACM Trans. Appl. Percept. 4, 2, 9.

Ledda, P., Chalmers, A., Troscianko, T., and Seetzen, H. 2005. Evaluation of tone mapping operators using a high dynamic range display. ACM Trans. Graph. 24, 3, 640-648.

Levinshtein, A., Stere, A., Kutulakos, K. N., Fleet, D. J., Dickinson, S. J., AND SiddiQI, K. 2009. Turbopixels: Fast superpixels using geometric flows. IEEE Trans. Pattern Anal. Mach. Intell. 31, 12 (Dec.), 2290-2297.

Lischinski, D., Farbman, Z., Uyttendaele, M., And SZELISKI, R. 2006. Interactive local adjustment of tonal values. ACM Trans. Graph. 25, 3, 646-653.

Mertens, T., Kautz, J., and Reeth, F. V. 2007. Exposure fusion. In Proceedings of Pacific Graphics 2007, IEEE, vol. 24, 382-390.

Pattanaik, S. N., Tumblin, J., Yee, H., and Greenberg, D. P. 2000. Time-dependent visual adaptation for fast realistic display. In Computer Graphics (SIGGRAPH '00 Proceedings), Addison Wesley, 47-54.

Pearson, E. S., And Hartley, H. O. 1988. Biometrika tables for statisticians 3 rd ed., vol. 1. Cambridge University Press.

Reinhard, E., Stark, M., Shirley, P., and Ferwerda, J. 2002. Photographic tone reproduction for digital images. In Proceedings of SIGGRAPH 2002, 267-276.

Reinhard, E., Heidrich, W., Debevec, P., Pattanaik, S., WARD, G., AND MyszKowski, K. 2010. High Dynamic Range Imaging: Acquisition, Display and Image-Based Lighting, second edition ed. Morgan Kaufmann, May.

REN, X., AND MALIK, J. 2003. Learning a classification model for segmentation. In Proc. 9th Int'l. Conf. Computer Vision, vol. 1, $10-17$.

Tumblin, J., And Rushmeier, H. E. 1993. Tone reproduction for realistic images. IEEE Computer Graphics and Applications 13, 6 (Nov.), 42-48.

Tumblin, J., Hodgins, J. K., And Guenter, B. K. 1999. Two methods for display of high contrast images. ACM Trans. Graph. $18,1,56-94$.

Ward Larson, G., Rushmeier, H., And Piatko, C. 1997. A Visibility Matching Tone Reproduction Operator for High Dynamic Range Scenes. IEEE Transactions on Visualization and Computer Graphics 3, 4 (Oct.), 291-306.

Yee, Y. H., And Pattanaik, S. 2003. Segmentation and adaptive assimilation for detail-preserving display of high-dynamic range images. The Visual Computer 19, 7-8.

Yoshida, A., Blanz, V., Myszkowski, K., And Seidel, H.P. 2005. Perceptual evaluation of tone mapping operators with real-world sceness. 192-203. 